Note

\title{
Tributyltin and mitochondria: new evidence in support of an uncoupling mechanism and a further characterisation of the transport mechanism
}

\author{
Marcantonio Bragadin ${ }^{\mathrm{a}, *}$, Daniele Marton ${ }^{\mathrm{b}}$, Sabrina Manente $^{\mathrm{a}}$, Guido Scutari ${ }^{\mathrm{c}}$, \\ Antonio Toninello ${ }^{\mathrm{c}}$ \\ a Dipartimento di Scienze Ambientali, Università di Venezia, DD 2137, I-30123 Venice, Italy \\ ${ }^{\mathrm{b}}$ Dipartimento di Chimica Inorganica, Metallurgia ed Analitica, Università di Padova, via Marzolo 1, 35131 Padua, Italy \\ ${ }^{\mathrm{c}}$ Dipartimento di Chimica Biologica, Università di Padova, e Centro Studio delle Biomembrane, C.N.R. Padova, Viale G. Colombo 3, 35121 Padua, \\ Italy
}

Received 15 July 2002; accepted 6 November 2002

\begin{abstract}
New evidence is given to support the hypothesis that the tributyltin compound (TBT) is an uncoupler of the oxidative phosphorylation in mitochondria. By means of an Arrhenius plot, it has been demonstrated that the transport kinetic of the TBT compound in mitochondria behaves in a manner which is similar to that obtained using classical uncouplers. The break point at $17^{\circ} \mathrm{C}$ observed in the Arrhenius plot is interpreted as due to a phase change in the lipidic bilayer, since the TBT compound, like uncouplers and mobile carriers, is sensitive to the phospholipidic phase change. Therefore, we can confirm our previous hypothesis that, as TBT is an uncoupler it, like uncouplers, crosses the biological membranes by means of an electrophoretic mechanism. An understanding of this behaviour is essential when an explanation of the neurotoxicity of TBT is required.
\end{abstract}

(C) 2002 Elsevier Science B.V. All rights reserved.

Keywords: Mitochondria; Tribuytltin; Uncoupling

\section{Introduction}

The toxicity of trialkyltin (TAT) compounds in humans and whole organisms has already been largely documented [1-12]. Many 'in vitro' experiments have been performed in cells and in subcellular structures in order to find the molecular mechanism which is responsible for this toxicity in animals. In particular, the interactions of TAT compounds with mitochondria, which are the energy source for the cell, have been widely studied [3-11]. All the results indicate that the TAT compounds inhibit ATP synthesis in mitochondria, but researchers do not agree about the mechanism responsible for the inhibition of the ATP synthesis. In particular, Aldridge et al. [3,4,6,7], suggest that TAT

\footnotetext{
* Corresponding author. Tel.: +39-041-234 8507; fax: +39-041-234 8584.

E-mail address: bragadin@unive.it (M. Bragadin).
}

compounds behave as $\mathrm{Cl}^{-} / \mathrm{OH}^{-}$exchangers in mitochondria, since they enter the membrane as alkyl ${ }_{3} \mathrm{SnCl}$ and are extruded as alkyl ${ }_{3} \mathrm{SnOH}$. The consequence of this cyclic mechanism is the collapse of the internal $\mathrm{pH}$ in mitochondria. More recently, Bragadin et al. [10-12] have proposed that the TAT compounds enter the mitochondria as alkyl$_{3} \mathrm{Sn}^{+}$aquo-cations and are extruded as alkyl $_{3} \mathrm{SnOH}$ electroneutral compounds. The balance of this cyclic mechanism is the transport, at any cycle, of a proton through the membrane, with a consequent $\Delta \mathrm{pH}$ and $\Delta \Phi$ collapse in the membrane. Since $\Delta \mathrm{pH}$ and $\Delta \Phi$ are the protonmotive force [13] which drives the ATP synthesis, this mechanism, which is similar to that induced by uncouplers (i.e. chemical compounds such as 2,4 dinitrophenol or FCCP which enhance proton permeability), could explain the ATP synthesis inhibition induced by TAT compounds. This last mechanism, however, could explain not only the toxicity, but, in particular, the neurotoxicity of TAT 
compounds. In fact, an uncoupling mechanism 'necessarily' implies a step in which the uncoupler (which must be a weak acid) crosses the membrane as a charged ion [13]; in the case of TAT compounds, the charged ion is the cation alkyl $_{3} \mathrm{Sn}^{+}$and the driving force for the transport of the cation is the negative-inside potential. Since nerve cells possess the highest (negative-inside) potential, and since the uptake rate depends exponentially on the applied potential [14], as already suggested by Bragadin et al. [10-12], this fact could explain why nerve cells are the preferential target for TAT compounds.

In this paper we give new evidence regarding this mechanism, resulting from the study of the transport mechanism of the tributyltin (TBT) compound at different temperatures. In fact, the aim of the paper is to confirm the uncoupler-like behaviour of TBT and to demonstrate that this compound crosses the membrane through the lipidic bilayer, in the same way as uncouplers, which are mobile carriers, and not by means of physiological carriers. These letters are not mobile carriers, but proteic pores (for example, the $\mathrm{Ca}^{2+}$ carrier, $[15,16])$ which connects the inside and outside aqueous phases in biological vesicles. This clarification is important since, if the transport of TBT occurs through a specific proteic pore, it becomes essential to find the particular protein present only in the nerve cells as a (specific) carrier of the TBT compound, in order to explain the neuroselectivity. On the contrary, if the transport mechanism occurs through the phospholipidic bilayer (as in the case of mobile carriers), this does not imply the presence of any particular carrier-protein in the nerve cells, since the phospholipidic bilayer has the same physical characteristics in all biological membranes.

\section{Materials and methods}

Mitochondria extracted from rat liver were prepared following the usual procedures [15]. The mitochondrial protein concentration was determined using the Lowry et al. method [17]. The potassium efflux was determined using a (Beckman) potassium selective electrode stored in a thermostated glass vessel with magnetic stirring. The recording apparatus was a Radiometer $\mathrm{pH}$ meter connected to a Texas recorder.

All the chemicals were of the highest purity commercially available. The valinomycin and FCCP (carbonylcyanide $p$-trifluoromethoxyphenulhydrazone) were obtained from Sigma. Tributyltin chloride was obtained from Aldrich.

The cyclosporin A was kindly donated by Professor P. Bernardi.

\section{Results and discussion}

The mitochondrial matrix contains about $0.1 \mathrm{M}$ potassium. If non-respiring mitochondria are resuspended in a potassium-free medium (or in a medium with a low potassium concentration), $\mathrm{K}^{+}$tends to efflux from the matrix to the resuspending medium, providing the presence of the potassium carrier, valinomycin, since the mitochondrial membrane is not permeable to the potassium ion (and to the protons). However, the $\mathrm{K}^{+}$ efflux is very limited, since it gives rise to an equivalent negative-inside potential gradient $(\Delta \Phi)$ (Fig. 1(a)). As $\Delta \Phi$ is an oppositive force, the extent of this force can easily be calculated and measured from the $\mathrm{K}^{+}$gradient $\Delta \Phi=\mathrm{RT} / n \mathrm{FLog}\left[\mathrm{K}^{+}\right]_{\text {in }} /\left[\mathrm{K}^{+}\right]_{\text {out }}$.

A massive and complete $\mathrm{K}^{+}$efflux occurs if a permeant positive counterion is added to the mitochondrial suspension. Fig. 1(b) shows that the addition of $\mathrm{Ca}^{2+}$ which enters the mitochondria through a selective channel [15,16] (Fig. 1(a)), induces a fast $\mathrm{K}^{+}$efflux since the positive charge of $\mathrm{Ca}^{2+}$ enters and neutralises the negative-inside potential. Analogously, if an uncoupler (FCCP) which permeates the membrane to the protons, is added, it induces a $\mathrm{K}^{+}$release (Fig. 1(b)). In this case, the efflux of $\mathrm{K}^{+}$is due to the same mechanism. This experimental condition (shown in Fig. 1) allows for a study of the transport kinetics of $\mathrm{K}^{+}$(by means of valinomycin) or $\mathrm{H}^{+}$(by means of uncouplers) if the respective transport is the rate limiting step in the whole kinetic. If valinomycin is the rate limiting step (i.e., a low valinomycin concentration and a high uncoupler concentration), the $\mathrm{K}^{+}$efflux measured, using a selective $\mathrm{K}^{+}$electrode, indicates the rate of transport of $\mathrm{K}^{+}$by means of valinomycin. If the proton transport is the rate limiting step (i.e., a low uncoupler concentration and a high valinomycin concentration), the $\mathrm{K}^{+}$efflux monitored by means of the $\mathrm{K}^{+}$electrode is a measure of the proton transport through the membrane.

Therefore, as discussed above, in order to measure the potassium transport, it is necessary to proceed using low valinomycin concentrations and a large amount of the uncoupler. As a means of verifying whether the potassium transport can be measured effectively under these conditions, a further measurement is performed after increasing the concentration of valinomycin. If the rate of $\mathrm{K}^{+}$efflux (measured as the initial rate, see Fig. 1b) is enhanced, this confirms that the valinomycin concentration is responsible for the limiting step in the condition under observation.

Analogously, in order to ensure that the proton transport is the limiting step under a particular experimental condition, it is sufficient to perform a successive measurement with a higher concentration of uncoupler. If the rate of $\mathrm{K}^{+}$efflux is enhanced, this demonstrates that the kinetics of the proton transport have been observed. 


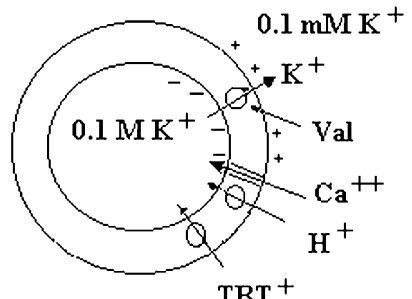

(a)

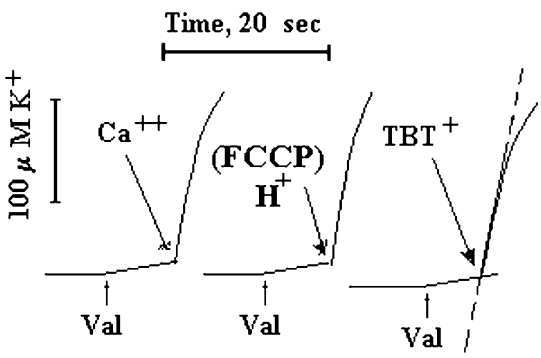

(b)

Fig. 1. The potassium efflux from the matrix of non-respiring mitochondria. The mitochondrial matrix contains $0.1 \mathrm{M}$ potassium. If the mitochondria $\left(0.2 \mathrm{mg} \mathrm{ml}^{-1}\right)$ are resuspended in a medium with a low potassium concentration (medium composition: $0.25 \mathrm{M}$ sucrose, $10 \mathrm{mM}$ HepesMops pH 7.4, $10 \mathrm{mM} \mathrm{MgCl} 2,100 \mu \mathrm{M} \mathrm{KCl}, 2 \mu \mathrm{M}$ rotenone, $1 \gamma$ cyclosporin A), a $\mathrm{K}^{+}$efflux occurs, providing the presence of valinomycin (Val) (2 $\mathrm{nmol} \mathrm{mg}{ }^{-1}$ protein), the potassium carrier (a). The $\mathrm{K}^{+}$efflux is very limited since the $\mathrm{K}^{+}$extrusion gives rise to an oppositive force: i.e. the negativeinside potential (a). A massive $\mathrm{K}^{+}$efflux occurs only if a positive counterion, e.g. $\mathrm{Ca}^{2+}$, (by means of the physiological carrier), or $\mathrm{H}^{+}$(by means of an uncoupler, FCCP), enters the mitochondria (b). In conditions of an excess of valinomycin $\left(1 \mathrm{nmol} \mathrm{mg}^{-1}\right.$ protein), the rate of $\mathrm{K}^{+}$efflux indicates the rate of $\mathrm{TBT}^{+}$transport (the initial rate is the slope of the straight line $\mathrm{K}^{+}$concentration vs. time, see the dotted line), when $2 \mu \mathrm{M}$ TBT (chloride) is added (b). $\mathrm{O}$, mobile carriers; =, proteic pore.

This procedure has been previously followed in order to study, by means of an Arrhenius plot, the transport kinetics of the mobile carriers (e.g. DNP, valinomycin) and the proteic pores such as the calcium carrier and gramycidin $[15,16]$. The results indicate that all pore carriers give an Arrhenius plot with a low slope, which corresponds to a low activation energy in the transport system, while all mobile carriers show two activation energies with a break point at $17^{\circ} \mathrm{C}[15,16]$. As the break point in the Arrhenius plot is the same concerning all mobile carriers, this was interpreted as due to a phase change in the lipidic bilayer. The failure of the break point observed in the $\mathrm{Ca}^{2+}$ and gramycidin transport was explained by the fact that a proteic pore is not sensitive to a phase change in the lipidic bilayer $[15,16]$.

Fig. 2 shows an Arrhenius plot in which the rate limiting step is the rate of transport of TBT. The graph is biphasic, showing two different activation energies, with a break point at $17^{\circ} \mathrm{C}$, as in the case of the uncouplers.

Since TBT in solution gives rise to the following equilibrium reactions [18]:

$$
\begin{aligned}
& \mathrm{Bu}_{3} \mathrm{SnCl}+n \mathrm{H}_{2} \mathrm{O} \rightarrow \mathrm{Cl}^{-}+\mathrm{Bu}_{3} \mathrm{Sn}\left(\mathrm{H}_{2} \mathrm{O}\right)_{n \text { aq }}^{+} \\
& \mathrm{Bu}_{3} \mathrm{Sn}\left(\mathrm{H}_{2} \mathrm{O}\right)_{n \text { aq }}^{+}+\mathrm{H}_{2} \mathrm{O} \\
& \rightleftarrows \mathrm{Bu}_{3} \mathrm{Sn}(\mathrm{OH})\left(\mathrm{H}_{2} \mathrm{O}\right)_{n-1(\mathrm{aq})}+\mathrm{H}_{3} \mathrm{O}^{+}
\end{aligned}
$$

the experimental data suggest that the TBT compound crosses the mitochondrial membrane as cation $\mathrm{Bu}_{3} \mathrm{Sn}\left(\mathrm{H}_{2} \mathrm{O}\right)_{n}^{+}$aq through the lipidic bilayer and not by making use of a physiological carrier.

This behaviour is an essential point in support of our hypothesis regarding the prevailing TBT neurotoxicity. In fact, our hypothesis, which is based on the presence in the nerve cells of the highest potential, which is the driving force for $\mathrm{Bu}_{3} \mathrm{Sn}\left(\mathrm{H}_{2} \mathrm{O}\right)_{n}^{+}$aq uptake [14], needs to be clarified with regard to the transport mechanism of TBT through the membrane.

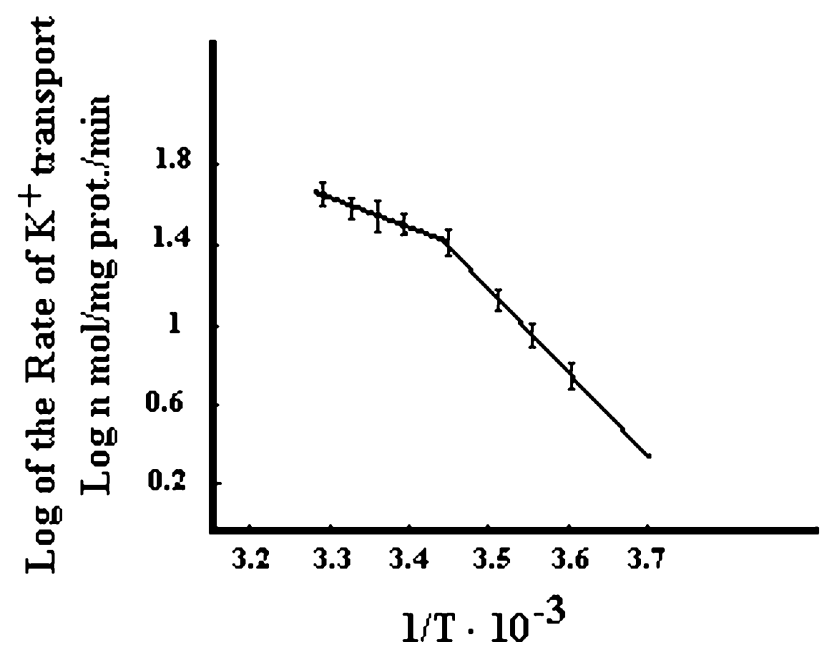

Fig. 2. Arrhenius plot for the transport of TBT in mitochondria. Medium composition: $0.25 \mathrm{M}$ sucrose, $10 \mathrm{mM}$ Hepes-Mops $\mathrm{pH}$ 7.4, 10 $\mathrm{mM} \mathrm{MgCl} 2,100 \mu \mathrm{M} \mathrm{KCl}, 2 \mu \mathrm{M}$ rotenone, $1 \gamma$ cyclosporin A. To the medium $(4 \mathrm{ml})$ containing the mitochondria, $\left(0.2 \mathrm{mg} \mathrm{ml}^{-1}\right)$ and the excess valinomycin ( $1 \mathrm{nmol} \mathrm{mg}^{-1}$ protein), $2 \mu \mathrm{M}$ TBT (chloride) was added and the (initial) rate of $\mathrm{K}^{+}$efflux was measured at different temperatures. The experimental points are the mean value obtained from five replicative measurements.

The transport can occur by means of two possible mechanisms:

a) specific carrier (proteic pore);

b) an electrophoretic transport through the lipidic bilayer.

As an explanation of neurotoxicity, the mechanism (a) implies that a specific carrier for $\mathrm{Bu}_{3} \mathrm{Sn}\left(\mathrm{H}_{2} \mathrm{O}\right)_{n}^{+}$aq should be present only in the nerve cells. Such a carrier has never been found, either in the mitochondrial, or in the cytoplasmic membrane. Furthermore, the biphasic 
behaviour of the Arrhenius plot excludes its presence in mitochondria and, in any case, it is difficult to justify the presence of a carrier for $\mathrm{Bu}_{3} \mathrm{Sn}\left(\mathrm{H}_{2} \mathrm{O}\right)_{n}^{+}$aq, which is not a physiological compound.

The electrophoretic transport mechanism (b) occurs through the lipidic bilayer which is the same in all biological membranes. In this case, the transport mechanism has no need of the presence of a carrier (a proteic pore) specific for a non-physiological compound.

Therefore our experimental data, are in agreement with a transport mechanism which is sensitive to the condition of the lipidic bilayer (mechanism b), and they are, therefore, indispensable in support of a characterisation of the neurotoxicity of TBT compounds.

\section{References}

[1] S.J. Blunden, P.A. Cusak, R. Hill (Eds.), The Industrial Uses of Tin Chemicals, Springer, Berlin, 1985.
[2] M.J. Selwin, in: V.G. Kummer Dos, N.G. Steik (Eds.), Chemistry and Technology of Silicon and Tin, Oxford University Press, New York, 1997.

[3] W.N. Aldridge, J. Biochem. 69 (1958) 367.

[4] W.N. Aldridge, B.W. Street, D. Skilleter, J. Biochem. 168 (1977) 353.

[5] D.N. Skilleter, J. Biochem. 146 (1975) 465.

[6] W.N. Aldridge, B.W. Street, J. Biochem. 91 (1964) 287.

[7] W.N. Aldridge, B.W. Street, J. Biochem. 124 (1971) 221

[8] I. Mellman, R. Fuchs, A. Helenius, Ann. Rev. Biochem. 55 (1986) 663.

[9] E.J. Bowman, J. Biol. Chem. 258 (1983) 15238.

[10] M. Bragadin, D. Marton, J. Inorg. Biochem. 68 (1997) 75.

[11] M. Bragadin, D. Marton, G. Scutari, P. Dell'Antone, J. Inorg. Biochem. 78 (2000) 205.

[12] M. Bragadin, D. Marton, A. Toninello, E.R. Viola, Inorg. Chem. Commun. 3 (2000) 255.

[13] P. Mitchell, Science 286 (1979) 1148.

[14] P. Lauger, Science 178 (1972) 24-30.

[15] M. Bragadin, T. Pozzan, G.F. Azzone, Biochem. 18 (1979) 5972.

[16] M. Bragadin, T. Pozzan, G.F. Azzone, FEBS Lett. 104 (1979) 347.

[17] O.H. Lowry, N.J. Rosembrough, A.L. Farr, R.J. Randall, J. Biol. Chem. 193 (1951) 265.

[18] R.S. Tobias, Organomet. Chem. Rev. 1 (1966) 93. 